\title{
¿Una intervención educativa en niños de doce años de Madrid modifica sus conocimientos y hábitos de higiene buco-dental?
}

\author{
Caldés Ruisánchez S*, Cea Sánchez N*, Crespo Aliseda P*, Díez Nicolás V*, \\ Espino García A*, Galán Arévalo $S^{*}$, Albaladejo Vicente R**, \\ Domínguez Rojas V**.
}

\section{RESUMEN}

Una adecuada educación en higiene buco-dental en niños es deseable para que éstos adquieran desde la infancia unos hábitos saludables que prevengan posibles enfermedades odontológicas en el futuro. En este estudio nos hemos propuesto valorar la eficacia de un programa de intervención en escolares que les proporcione unos conocimientos básicos sobre este aspecto sanitario. Para ello hemos impartido a sesenta niños y niñas de sexto de primaria (de doce años) del colegio Berriz Veracruz una charla educativa, distribuyéndoles dos encuestas, una antes y otra cuatro semanas después del programa educativo, y hemos estudiado los conocimientos y hábitos que sobre salud e higiene buco-dental presentaron antes de la intervención, así como las modificaciones que sufrieron tras la misma.

Observamos cambios significativos tanto en la frecuencia como en la técnica de cepillado, en la actitud a tomar ante el sangrado de encías y en el abandono de hábitos nocivos.

Por todo ello concluimos que los programas educativos sobre higiene y salud buco-dental son eficaces para modificar positivamente las conductas de niños de esta edad, subrayando el interés que tiene la implantación de los mismos en el ámbito escolar.

Palabras clave: Salud bucodental, niños, intervención educativa..

\section{ABSTRACT}

The proper education on dental hygiene is desirable for children, so that they acquire from an early age good habits that prevent possible future diseases. Our study focuses on the results of an educational program for school age children that provide basic knowledge about dental hygiene. To do this, we have given an educational workshop to a group of sixth graders from The School Berriz Veracruz. We conducted a survey before and after the survey to monitor the children's dental hygiene habits before and after the workshop. That way we could determine the changes in attitudes upon attending an information workshop on dental hygiene.

We noticed significant changes both in the frequency and technique of mouth washing. We also observed changes in attitude toward gums bleeding and abandonment of poor hygiene habits.

Thus, we conclude that educational workshops about dental hygiene provide positive results in children's habits. We must highlight the importance of implementing such workshops among school-age children.

Key words: Dental hygiene, children, educational workshops. 
Aceptado para publicación: Octubre 2004.

* Alumnos de sexto curso de Medicina.

** Departamento de Medicina Preventiva, Salud Pública. Facultad de Medicina UCM. Madrid.

Caldés Ruisánchez S, Cea Sánchez N, Crespo Aliseda P, Díez Nicolás V, Espino García A, Galán Arévalo S, Albaladejo Vicente R, Domínguez Rojas V. ¿ Una intervención educativa en niños de doce años de Madrid modifica sus conocimientos y hábitos de higiene buco-dental? Av. Odontoestomatol 2005; 21-3: 149-157.

\section{INTRODUCCIÓN}

En la educación para la salud participan varias disciplinas claramente diferenciadas como las ciencias de la salud, de la conducta, de la educación y de la comunicación, que serán el núcleo básico sobre el que se sustenta el programa tanto teórico como práctico de la Educación para la Salud (1).

La aparición de muchas enfermedades que nos afectan en la actualidad es debida al inadecuado estilo de vida y a los hábitos insanos adquiridos a lo largo del tiempo (2). El mecanismo más eficaz que tenemos en la actualidad, para generar en las personas un cambio de actitud hacia modos de vida más saludables, es la Educación para la Salud, en todos sus ámbitos de actuación, preservando siempre el derecho fundamental de las personas de obrar en libertad, sin imposición por parte de los educadores (1).

La introducción en el programa escolar de la Educación para la Salud buco-dental es de una importancia trascendental. Temas como la caries (3) y sus técnicas de prevención $(4,5,6,7,8)$, la enfermedad periodontal, las técnicas de cepillado, el uso de seda dental, las maloclusiones dentarias y las revisiones periódicas por el Odontólogo $(9,10,11)$, son de obligado tratamiento en el ámbito escolar, hasta conseguir la adquisición de hábitos saludables que trasciendan al entorno familiar de los escolares y que perduren en el tiempo.

La caries es la enfermedad crónica infecciosa más prevalente en el hombre $(11,12,13,14,15,16)$. La enfermedad periodontal se previene eliminando la placa bacteriana con un cepillado minucioso y evitando las maloclusiones dentarias, bien sean congénitas (aquellas provocadas por alteración del núme- ro, tamaño o forma de los dientes y por deformidades de los maxilares, y cuya resolución es la ortodoncia o la cirugía) o adquiridas (consecuencia de hábitos nocivos tales como el uso prolongado del chupete o biberón, chuparse el dedo, morderse las uñas u otros objetos, respirar por la boca). La prevención de éstas consiste fundamentalmente en el destierro de dichos hábitos nocivos (10) y un número adecuado de visitas periódicas al odontólogo $(11,17)$.

Por todo ello, en la realización de este estudio se ha creído oportuno llevar a cabo una intervención en escolares de doce años utilizando un método directo de educación, la charla, como herramienta fundamental de trabajo. Además se valoraron sus conocimientos previos y se compararon con los que manifestaron después de la misma. La finalidad es demostrar que una educación correcta y exhaustiva sobre salud buco-dental en alumnos de sexto de primaria (doce años) mejora sus hábitos higiénicos, y que en edades tempranas esta actuación es más efectiva puesto que todavía su conducta es modificable.

\section{OBJETIVOS}

1. Conocer la situación sobre conocimientos de salud buco-dental y hábitos de higiene en escolares de sexto de primaria (doce años), y analizar los diferentes factores que influyen en los mismos.

2. Realizar una intervención educativa sobre salud buco-dental en dichos escolares.

3. Analizar las posibles modificaciones de los conocimientos y hábitos higiénicos a raíz de la intervención mencionada. 


\section{MATERIAL Y MÉTODOS}

Se ha realizado un estudio cuasiexperimental de tipo serie temporal, usando el mismo grupo, escolares de sexto de primaria, como control (antes de la intervención) y como experimental (después de la intervención).

Nuestro trabajo consta de dos fases:

1. Con el permiso de la dirección y la Asociación de Padres de Alumnos (A.P.A.) del Colegio Bérriz Veracruz de Molino de la Hoz (Las Rozas, Madrid), impartimos una charla $(11,12,18,19)$ sobre salud buco-dental adaptada a escolares, en una población de 60 niños (31 niños y 29 niñas) de sexto de primaria, distribuidos en dos clases. Se recogieron los datos del total de los niños que participaron en el estudio en ambos casos. El material audiovisual utilizado fue elaborado por el Ministerio de Sanidad y Consumo $(11,20)$.

2. Distribuimos en esta población dos encuestas. En la primera de ellas, previa a la intervención, se consideraron las siguientes variables $(21,22,23,24)$.

- Edad.

- Sexo.

- Hermanos mayores en la familia, para valorar la posible influencia de éstos en las costumbres de higiene y salud buco-dental.

- Frecuencia de visitas al dentista (25).

- Uso actual o pasado de ortodoncia, analizando si un contacto directo con el odontólogo y la enfermedad buco-dental influye positivamente en el niño.

- Hábitos de higiene bucodental (26): número de cepillados al día, cuidado de las encías sangrantes, uso de enjague bucal y dentríficos fluorados $(27,28,29)$.

- Hábitos nocivos: respiración bucal, chuparse el dedo, morderse las uñas, ausencia de cepillado de dientes inmediatamente posterior a la ingesta de azúcar.

La segunda encuesta se pasó cuatro semanas después de la intervención. Aparte de los ítems incluidos en la anterior, en ella se añadieron las siguientes cuestiones $(10,11,12)$ :

- Comentario a la familia de la charla educativa, estudiando si este hecho está relacionado con la asimilación de los hábitos que se proponen en la misma.

- Adquisición de hábitos higiénicos a raíz de la intervención.

- Modificación de los posibles hábitos nocivos.

En la elaboración y procesamiento de los datos se utilizó el programas SPSS, y en el análisis de las variables empleamos pruebas Chi cuadrado y ANOVA, tomando como nivel de significación $p<$ 0,05 .

\section{RESULTADOS}

En la población total de 60 alumnos de sexto de primaria (31 niños y 29 niñas de doce años de edad) se obtuvieron los siguientes resultados tras el análisis de los datos, antes y después, de la intervención educativa.

\section{HERMANOS MAYORES EN LA FAMILIA}

El $61.7 \%$ de los encuestados tenía hermanos mayores, frente a un $38.3 \%$ que no los tenía. No se encuentran diferencias signicativas en lo que concierne a sus hábitos de higiene.

\section{VISITAS AL DENTISTA}

En la primera encuesta se observa que la mayoría de los niños acude al dentista al menos una vez al año (58,3\%), frente a un grupo que lo hace muy frecuentemente, es decir, más de tres veces al año (35\%). Esta respuesta fue incluida en la encuesta para cribar a los alumnos que llevaran ortodoncia, ya que éstos tendrían una frecuencia muy elevada. Tan sólo un $6,7 \%$ no visita al dentista regularmente. No se comprueba ninguna asociación estadística entre esta variable y el resto de la encuesta.

Tras la intervención se preguntó con qué frecuencia creen ellos que se debe ir al dentista, y en este caso un $54,2 \%$ pensó que hay que ir al menos una vez al año, mientras que un 45,8\% opinaba que sólo era 


\begin{tabular}{|lccc|}
\hline $\begin{array}{l}\text { TABLA 1.- MEDIA, AMPLITUD DE VARIACIÓN E INTERVALO DE CONFIANZA (IC 95\%) DEL NÚMERO } \\
\text { DE UNIDADES FORMADORAS DE COLONIAS (EN LOG) ANTES Y DESPUÉS DEL LAVADO CON } \\
\text { SOLUCIÓN DE HIDRÓXIDO DE CALCIO PARA LAS } 30 \text { MUESTRAS. NATAL / RN - 2003 }\end{array}$ \\
\hline \multicolumn{1}{|c|}{$\begin{array}{l}\text { Momentos } \\
\text { Media }\end{array}$} & Desuío patrón & IC (95\%) \\
\hline Antes del lavado & 1.08 & $0-3.53$ & $0.59-1.58$ \\
\hline Después del lavado & 0.67 & $0-2.60$ & $0.30-1.03$ \\
\hline $\begin{array}{l}\mathrm{p}=\mathbf{0 , 0 9 3 4} \\
\text { Fuente: (Programa de post grado en ciencias de la salud) }\end{array}$ & & \\
\hline
\end{tabular}

\begin{tabular}{|c|c|c|c|}
\hline Momentos & Media & Desuío patrón & IC (95\%) \\
\hline Antes del lavado & 2.54 & $1.69-3.53$ & $2.17-2.84$ \\
\hline Después del lavado & 1.69 & $0.00-2.30$ & $0.44-1.73$ \\
\hline \multicolumn{4}{|l|}{$p=0,0007$} \\
\hline Fuente: (Programa de post & a salud) & & \\
\hline
\end{tabular}

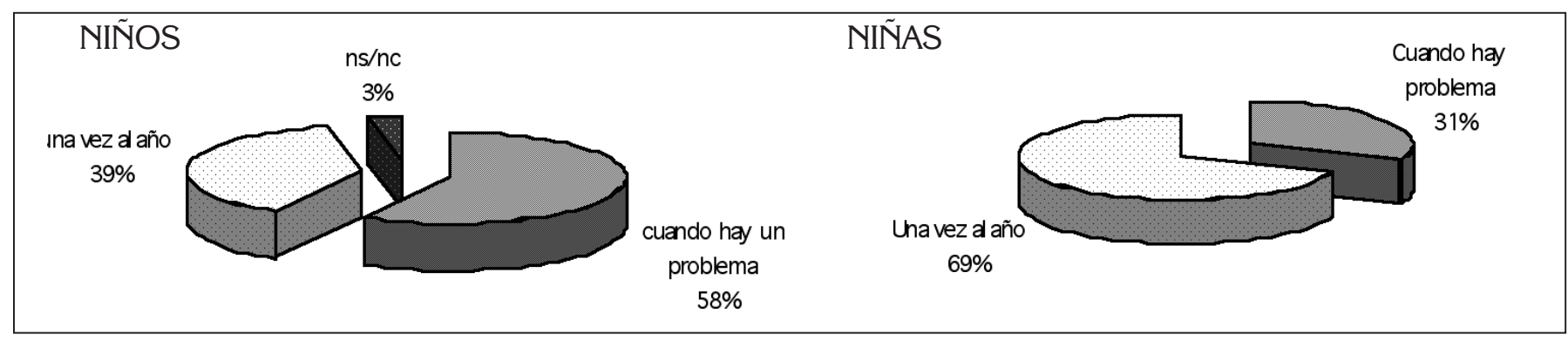

Figura 1. Diferencia por sexos a la respuesta de "cuándo creían que debían ir al dentista".

necesario ir cuando existía algún problema. Se obtuvo significación estadística por la variable sexo, en el sentido de que las niñas son mayoría dentro de los del primer grupo $(62,5 \%)$, y los niños dentro del segundo (66\%), (Figura 1). También se observó un cambio, no significativo estadísticamente pero que si apunta una tendencia, en el sentido de que en la segunda encuesta, el porcentaje de los que creían necesario ir al menos una vez al año al dentista era mayor que el porcentaje de niños que en la primera encuesta declararon ir con esa frecuencia.

Al contrario de lo esperado, no ha sido estadísticamente significativo la asociación entre la frecuencia de visitas al dentista con el número de cepillados al día, ni con el uso de cepillos especiales para niños.

\section{COMENTARIO DE LA CHARLA}

Tras impartir la charla, el $85 \%$ afirmaba que la comentó con su familia. Un $54,9 \%$ de éstos fueron niñas, resultando la diferencia por sexos estadísticamente significativa.

Aquellos que comentaron la charla habían modificado su técnica de cepillado en mayor medida que el resto de niños, de forma significativa. 

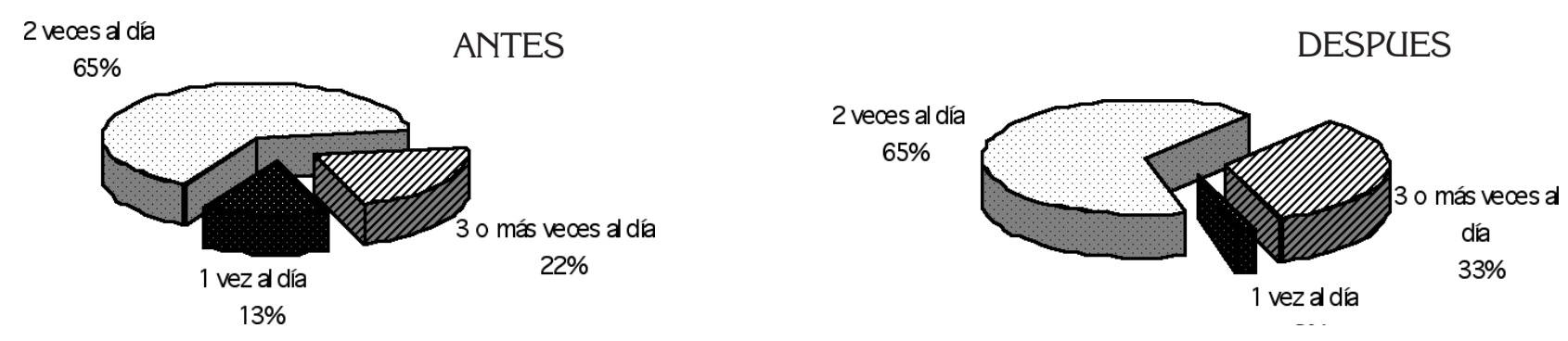

Figura 2. Frecuencia de cepillados / día antes y después de la intervención educativa.

\section{ORTODONCIA}

El 28,3\% de los escolares del estudio llevaban o habían llevado ortodoncia y, como es evidente, en este grupo había una inmensa mayoría que acudía al dentista muy frecuentemente (88\%).

Sin embargo sorprende que el $75 \%$ de ellos, creía que sólo deberían ir al dentista cuando surgía algún problema. Este dato es estadísticamente significativo, es decir, los niños que llevaban o habían llevado ortodoncia creían menos importante acudir al dentista con una frecuencia determinada que los que no la habían llevado nunca (un 65,11\% de éstos que no la habían llevado nunca opinaba que debía ir al menos una vez al año). Asimismo, los que habían llevado ortodoncia se cepillaban menos los dientes tras la ingesta de dulces que los que no la habían llevado, de una manera estadísticamente significativa. No se comprende bien esta asociación, y puede deberse a la existencia de algún sesgo que debería ser estudiado con mayor detenimiento.

En cambio no hay asociación entre el número de cepillados al día y el haber llevado ortodoncia. Esperábamos que aquellos que habían llevado ortodoncia se cepillarían los dientes con más frecuencia del resto por su mayor contacto con el dentista.

\section{CEPILLADOS AL DÍA}

En la primera encuesta se observa que la mayoría de los niños se cepillaba dos veces al día (65\%), mientras que un $21,7 \%$ se cepillaba tres o más veces al día. Tan sólo un 13,3\% lo hacía una vez al día.

Ninguno de los niños del estudio se cepillaba en el colegio, pero esto no se ha visto modificado tras la intervención. Durante la intervención los niños comentaron la imposibilidad de cepillarse los dientes tras la comida en su colegio. Consideramos este dato especialmente importante, ya que demuestra la escasa implicación de los centros en la generalización de estas conductas saludables.

Comparando los resultados con la segunda encuesta, un $73,3 \%$ afirmaba que se cepillaba más veces al día que antes de la charla. Dentro de éstos había una mayoría que había hablado con su familia de la intervención (90,9\%). Ambos resultados son estadísticamente significativos. Es decir, globalmente los niños confesaban haber aumentado la frecuencia de cepillado a raíz de la charla, pero aquellos niños que comentaron la charla con su familia demuestran un incremento más notable que el resto.

Además, comparando los resultados objetivos obtenidos a la pregunta "cंcuántas veces te cepillas los dientes al día?” (repetida en ambas encuestas), se comprueba un aumento estadísticamente significativo en la frecuencia de cepillados. Es decir, no sólo lo afirman sino que esta afirmación se corresponde con los datos reales obtenidos (Figura 2).

En cuanto a la técnica de cepillado, un $81,7 \%$ afirmaba haberla modificado a raíz de la charla, y este resultado se ve incrementado, como ya se ha señalado, dentro del grupo de los que habían comentado la intervención con su familia. Los dos resultados tienen significación estadística.

\section{SANGRADO DE ENCÍAS}

En la primera encuesta la mayoría de los niños decí- 
an cepillarse mejor los dientes cuando les sangraban las encías (49,1\%), y este porcentaje aumenta en la segunda encuesta significativamente $(73 \%)$.

\section{UTILIZACIÓN DE CEPILLO ESPECIAL PARA NIÑOS}

Un 30,9\% de los niños utilizaba un cepillo adaptado a su edad en la primera encuesta, y en la segunda este grupo asciende a 47,3\%, mostrándose una tendencia al cambio.

\section{HÁBITOS NOCIVOS}

Se preguntó en ambas encuestas a los niños si tenían hábitos nocivos. En la primera encuesta respondió afirmativamente un $66,7 \%$, mientras que en la segunda lo hizo un $76,7 \%$. Este incremento es llamativo, y puede deberse a que la primera vez desconocían cúales eran los hábitos nocivos entre aquellos que realizaban.

En la segunda encuesta el $56 \%$ de los que tenían dichas conductas, afirmó haberlas abandonado, mientras que un $44 \%$ no lo había hecho. Sin embargo, el 58,3\% de aquellos que no lo habían hecho se proponían intentarlo.

\section{OTRAS VARIABLES}

En cuanto al resto de variables estudiadas no hubo oscilaciones importantes de las mismas, ni se obtuvo significación estadística al compararlas con el resto de los items. Los porcentajes que reflejaron se recogen en la Tabla 1:

En cuanto al consumo de dulces los resultados obtenidos se muestran en la Tabla 2.

\section{DISCUSIÓN Y CONCLUSIONES}

El método de intervención empleado, a nuestro juicio, ha sido adecuado, teniendo en cuenta tanto el cambio de conocimientos y conductas que pretendíamos, como los recursos de los que disponíamos en el momento en el que realizamos el trabajo. El hecho de haber apoyado la charla educativa que dirigimos a los escolares en diapositivas con dibujos adaptados a su edad, se ha revelado como un método eficaz para transmitirles los conocimientos básicos que pretendíamos en un principio.

Además de la charla, mostramos de forma dinámica e interactiva la técnica de cepillado recomendada por los expertos en la actualidad, como soporte fundamental al material audiovisual.

\begin{tabular}{|c|c|c|}
\hline \multicolumn{3}{|c|}{ TABLA 1.- OTRAS VARIABLES } \\
\hline VARIABLE & FRECUENCIA antes (\%) & FRECUENCIA (después) \\
\hline Pasta con fluor & 91,1 & 96,6 \\
\hline Uso de colutorios & 44,1 & 54,2 \\
\hline Colutorio fluoorado & 69,4 & 67,9 \\
\hline
\end{tabular}

\begin{tabular}{|c|c|c|}
\hline \multicolumn{3}{|c|}{ TABLA 2.- CONSUMO DE DULCES } \\
\hline & PRIMERA ENCUESTA (\%) & SEGUNDA ENCUESTA \\
\hline A diario & 11,7 & 3,3 \\
\hline Todas las semanas & 21,7 & 28,3 \\
\hline Ocasionalmente & 66.7 & 693 \\
\hline
\end{tabular}


En la elaboración de la intervención nos habría gustado disponer de productos de higiene buco-dental (revelador de placa, cepillos pediátricos, colutorios y dentífricos fluorados) con el fin de que se familiarizaran los niños con los mismos. Además considerábamos que este tipo de obsequios favorecerían un mayor interés y recuerdo de la charla por parte de los niños. Sin embargo, tal intención se hizo inviable por el desinterés mostrado por la industria especializada del sector a la que recurrimos.

Por otro lado, como limitación de este estudio consideramos necesario señalar que en la intervención no pudimos valorar la técnica de cepillado de cada uno de los escolares, así como corregir individualmente los posibles defectos en la misma. En este sentido, también pensamos que hubiera sido interesante añadir a las encuestas métodos de comprobación objetiva de los cambios que los niños manifestaron en la segunda encuesta (reveladores de placa y observación directa del cepillado (9)), tal y como se ha realizado en otros estudios y que hubiera conferido mayor fiabilidad a los datos (18).

Asimismo, un mayor intervalo entre la intervención educativa y la segunda encuesta, asociado a un seguimiento en la evolución de los hábitos higiénicos más estrecho y continuado, podría haber contribuido a mejorar los resultados obtenidos, razón por la que creemos que estos aspectos se deben tener en cuenta en trabajos futuros.

Por otra parte, opinamos que la edad de los escolares incluidos en el estudio es adecuada para aprender una conducta de higiene óptima, puesto que los hábitos nocivos en la mayoría de ellos no están aún instaurados y, en caso de estarlo, es más sencillo modificarlos (9). Además sería interesante estudiar la necesidad de dar charlas de recuerdo periódicamente, analizando su utilidad y la frecuencia más adecuada.

Tenemos que reseñar que la actitud receptiva y la atención mostrada por los escolares fue muy satisfactoria. Además, la respuesta de los profesores en todo momento sobrepasó nuestras expectativas iniciales, lo cual contribuyó sobremanera a la calidad de la intervención.

Este trabajo nos ha permitido concluir que en nues- tra población, las niñas han comentado más la charla con su familia, a la vez que han asumido en mayor proporción que deben acudir al menos una vez al año al odontólogo. Por otro lado, los que han comentado la charla son aquéllos que han modificado en mayor medida su técnica de cepillado.

Además, se puede afirmar que la intervención ha servido para:

- Aumentar la frecuencia de cepillado (pasaron de un $87 \%$ a un $98 \%$ los niños que se cepillaban más de dos veces al día).

- Aprender que ante el sangrado de encías se deben cepillar mejor (de un $49,1 \%$ a un $73 \%$ ). Modificar la técnica de cepillado (un $81,7 \%$ afirma haberlo hecho).

- $\quad$ Abandonar los hábitos nocivos o tener el propósito de hacerlo (un 56\% comentaban haberlos abandonado, y un 58,3\% de los que no lo habían hecho se proponían intentarlo).

A tenor de los datos obtenidos se puede concluir que una intervención educativa como la realizada, tanto en el contenido de la charla como en el grupo etario al que está dirigida (escolares de sexto de primaria), sirve para mejorar el conocimiento de estos niños sobre higiene y salud buco-dental, así como para modificar positivamente sus comportamientos. Esto concuerda con lo que otros estudios similares $(9,18,21,30)$.

Finalmente, proponemos que se le dé una mayor importancia a este tipo de intervenciones educativas y se incluyan en los contenidos de la programación escolar. Igualmente, consideramos oportuna una mayor implicación de la familia y los odontólogos. Asimismo, creemos que los colegios deberían involucrarse en que sus alumnos adquirieran unos hábitos higiénicos adecuados, facilitándoles los recursos necesarios para favorecer conductas saludables tales como un cepillado dental tras la comida.

\section{BIBLIOGRAFÍA}

1. Rafael Rioboo García. Educación para la Salud Bucodental. En: Avances. Odontología Preventiva y Odontología Comunitaria. Madrid, 2002; p. 1321-41. 
2. V. Domínguez Rojas; P. Astasio Arbiza; P. Ortega Molina; E. Gordillo Florencio; J.A. García Núñez and A. Bascones Martínez. Análisis of several risks factors envolved in dental caries trough multiple logistic regresión. International Dental Journal, 1993, 43: 149-56.

3. R. Rodríguez Contreras; C. Gómez Pérez; N. Fernández Crehuet; D. Jurado Chacón; R. Gálvez Vargas. Problema sanitario de la caries dental. Estudio epidemiológico en escolares de dos zonas de Granada. Atención Primaria, 1987, 4 (5): 252-7.

4. J. Grytten; I. Rosson; D. Holst; L. Steele; Longitudinal study of dental health behaviours and other caries predictors in early childhood. Comm Dent Oral Epidemiol, 1988, 16: 356-9.

5. U. Hölund. Relationship between diet-related behaviour and caries in a group of 14-years-old Danish children. Comm Dent Oral Epidemiol, 1987, 15: 184-7.

6. B. A. Burt; S. A. Ekluns; K. J. Morgan; F. E. Larkin; K. E. Guire; L. O. Brown; J. A. Weintraub. The effects of sugars intake and frecuency of ingestion on dental caries incremented in a three-year longitudinal study. J Den Res, 1988, 67 (11): 1422-9.

7. V. Dominguez Rojas; P. Astasio Arbiza; P. Ortega Molina; L. Pérez Bravo; A. De Pereda García; J. A. García Núñez. Caries dental: Edad, nivel de ingresos y atención odontológica. Avances en odontoestomatología, 1991, 7: 431-6.

8. M. E. Serra; G. Bosch; L. Beltrán; N. Jorba; C. A. González; A. Agudo. Efecto de la prevención de caries mediante enjuagues fluorados. Resultados de un ensayo de campo no randomizado a los 7 años de seguimiento. Rev San Hig Publications, 1990, 64: 547-60.

9. López Bermejo M.A.;González Sanz A.; Cerón Vivancos J.; Moranz M. Estudio piloto de un programa preventivo bucodental para la población escolar de Madrid. Rev. Esp. Estomatol, 1988, 4: 279-90.
10. Demographic Factors, denture status and oral health-related quality of life. Community Dent Oral Epidemiol. 2004 Apr; 32 (2): 125-32.

11. Dirección General de Salud Pública. Ministerio de Sanidad y Consumo. Material audiovisual: Salud Buco-dental.

12. Junta de Extremadura. Consejería de Bienestar Social. Dirección General de Salud Pública. Programa de Salud Buco-dental. Badajoz 1998.

13. Smyth Chamosa E.; Taracido Trunk M.; Gestal Otero J.J. En: Ediciones Díaz de Santos, S.A. El flúor en la prevención de la caries dental.

14. R. L. Ettinger; J. Jacobsen. Caries: a problem in and overdenture population. Dent Oral Epidemiol, 1990, 18: 42-5.

15. J. R. Villalbi; R. Rico; J. C. Pérez-Gaeta; J. Casanova. El problema de la caries dental en la edad escolar: un estudio transversal. Rev Hig San Pub, 1985, 59: 1215-22.

16. V. Domínguez Rojas; M. V. Acedo Díaz-Pache; P. Astasio Arbiza; P. Ortega Molina; L.A. Pérez Bravo; M. E. Calle Purón. Prevalencia de caries en una población escolar. Avances en Odontoestomatología, 1990, 6: 271-279.

17. Junta de Castilla y León. Consejería de Sanidad y Bienestar Social. Valladolid 1995. Estudio Epidemiológico de la Salud Bucodental en los Escolares de Castilla y León.

18. Monique G. Julien. The Effect of Behaviour Modification Techniques on Oral Hygiene and Gingival Health of 10-Year-Old Canadian Children. Int J Paediatr Dent, 1994 Mar, 4(1): p. 3-11.

19. Estudio Prospectivo Delphi Libro Blanco. Odonto-Estomatología 2005. Barcelona: Ed. Lácer SA, 1997.

20. Ministerio de Sanidad y Consumo. Encuesta Nacional de Salud. Madrid, 1989. p. 86-94. 
21. Caroline A. Redmon; Fiona A. Blinkhorn; Elizabeth J. Kay; Robin M. Davies; Helen V. Worthington; Anthony S. Blinkhorn. A Cluster Randomized Controlled Trial Testing the Effectiveness of a School-based Dental Health Education Program for Adolescents. J Public Health Dent, 1999, 59: 12-7.

22. Lopez Bermejo M.A.; Samara Shukeir G.; Reyes Serrano P.; Oteo Muñoz C.; Cerón Vivancos J. Encuesta sobre Hábitos, Actitudes y Conocimientos sobre Higiene Oral en Niños de 6-7 Años de Edad de la CAM. Odontol Pediatr, 2002, Vol. 10, No 1 , p. 9-20

23. Administración de la Comunidad Autónoma del País Vasco. Departamento de Sanidad y Consumo. Estudio Epidemiológico de la Salud Buco-dental infantil en la Comunidad Autónoma Vasca, 1990.

24. Generalitat Catalunya. Enquesta Epdemiológica de salud bucodental de Catalunya 1997. Informe preliminar. Direccio General de Salut Publica. Noviembre, 1997, 7-25.
25. V. Domínguez Rojas; P. Astasio Arbiza; P. Ortega Molina; M. E. Calle Purón; M. V. Acedo DíazPache; L. A. Pérez Bravo. Atención odontológica en niños de 6 a 15 años. Avances en Odontoestomatología, 1990, 6: 549-58.

26. K. A. A. S. Warnakulasuriya. Social factors and oral hygiene habits among caries free children in a low fluoride area in Sri Lanka. Community Dent Oral Epidemiol, 1988, 16: 212-4.

27. S. M. Szpunar; B. A. Buró. Dental caries, flourosis and fluoride exposure in Michigan schoolchildren. J Dent Res, 1988, 67 (5): 802-6.

28. A. J. Spencer. Past association of fluoride vehicles with caries severity in Australian adolescents. Comm Dent Oral Epidemiol, 1986, 14: 233-7.

29. J. P. Carlos. Fluoride mouthrinses. In: Clinical Uses of fluorides. Ed. S. H. Y. Wei. Philadelphia, Pa: Lea E Febiger, 1985, p. 75-82.

30. M. A. Tapias; G. De Miguel; R. Jiménez-García; A. González; V. Domínguez. Incidente of caries in an infant population in Móstoles, Madrid. Evaluation of a preventive program after 7.5 years of followup. Int J Paediatr Dent, 2001, 11 (6), p.440. 